\title{
Robot-assisted distal pancreatectomy improves spleen preservation rate versus laparoscopic distal pancreatectomy for benign and low-grade malignant lesions of the pancreas
}

\author{
Yabo Jiang ${ }^{1 \#}$, Kailian Zheng ${ }^{2 \#}$, Shichao Zhang ${ }^{1 \#}$, Zhuo Shao ${ }^{2}$, Peng Cheng ${ }^{2}$, Yijie Zhang ${ }^{2}$, Gang Jin ${ }^{2}$, \\ Tianlin $\mathrm{He}^{2}$ \\ ${ }^{1}$ Eastern Hepatobiliary Surgery Hospital, Second Military Medical University, Shanghai, China; ${ }^{2}$ Department of Pancreatic Surgery, Changhai \\ Hospital, Second Military Medical University, Shanghai, China \\ Contributions: (I) Conception and design: Y Jiang, K Zheng, T He; (II) Administrative support: G Jin, T He; (III) Provision of study materials \\ or patients: Z Shao, P Cheng, Y Zhang, G Jin; (IV) Collection and assembly of data: Z Shao, P Cheng, Y Zhang, G Jin; (V) Data analysis and \\ interpretation: Y Jiang, K Zheng, S Zhang; (VI) Manuscript writing: All authors; (VII) Final approval of manuscript: All authors. \\ \#These authors contributed equally to this work. \\ Correspondence to: Tianlin He. Department of Pancreatic Surgery, Changhai Hospital, Second Military Medical University, Shanghai, China. \\ Email: skyrainhe@163.com.
}

Background: The aim of this retrospective study was to compare robot-assisted distal pancreatectomy (RDP) with laparoscopic distal pancreatectomy (LDP) for patients with benign and low-grade malignant lesions of the pancreas.

Methods: This study included 166 patients who underwent RDP ( $\mathrm{n}=63)$ or LDP ( $\mathrm{n}=103)$ for benign or low-grade malignant lesions of the pancreas from January 2011 to October 2018 in Changhai Hospital. A retrospective analysis was performed between the two groups. The primary points were operation time, operative blood loss, hospital stay, pancreatic fistula and spleen preservation.

Results: There were no significant differences in patient characteristics or indications between RDP and LDP. Notably, among all patients, RDP was associated with a significantly higher rate of spleen preservation than that for LDP $(30.2 \%$ vs. $6.8 \%, \mathrm{P}<0.001)$, while other intraoperative variables were similar between the two groups. No death cases in the study group within 30 days were reported in either group. Referring to postoperative outcomes, postoperative pancreatic fistula (POPF) was 22\% for the RDP group and 33\% for the LDP group. In addition, the rate of clinically significant grade B/C pancreatic fistula was $5 \%$ and $7 \%$, respectively. There were also no significant differences in hospital stay $(6.0 \pm 3.0$ vs. $6.4 \pm 2.5, \mathrm{P}=0.404)$. Furthermore, the univariate analysis demonstrated that tumor size $(\mathrm{P}=0.001)$ and surgery group $(\mathrm{RDP} / \mathrm{LDP})$ $(\mathrm{P}=0.002)$ were associated with SP rate independent factor for spleen preservation.

Conclusions: RDP is an effective and safe technique with significant advantage in spleen preservation for patients with benign and low-grade malignant lesions in the distal pancreas.

Keywords: Robot-assisted distal pancreatectomy (RDP); laparoscopic distal pancreatectomy (LDP); spleen preservation

Submitted Oct 10, 2019. Accepted for publication Jul 24, 2020.

doi: $10.21037 /$ tcr-19-2121

View this article at: http://dx.doi.org/10.21037/tcr-19-2121 


\section{Introduction}

The frequency of minimally invasive (MIS) pancreatectomy has been increasing for both benign and malignant diseases. Among these surgeries, laparoscopic distal pancreatectomy (LDP) is the most accepted in the literature to date (1). Recent studies have demonstrated that the LDP approach is often recommended because of reduced blood loss and shorter hospital length of stay compared to the traditional open procedure $(2,3)$. Furthermore, since the first robotassisted pancreatic surgery was reported, approximately 200 robot-assisted distal pancreatectomies (RDPs) have been reported (4) with significant advantages of threedimensional visual fields and highly flexible functions (5). Additionally, despite technical difficulties and concern for oncologic outcomes versus other approaches, the choice of an RDP or LDP approach for distal pancreatectomy has remained a controversial topic $(6,7)$. A single-center retrospective study demonstrated that RDP is equally as effective and safe as conventional LDP, but RDP significantly reduced the risk of conversion to an open procedure even in the treatment of malignant diseases (8). Furthermore, the use of robots has also been reported to improve the spleen preservation (SP) rate compared to LDP $(9,10)$. The spleen should be preserved as much as possible especially in the case of benign or borderline diseases because SP can offer patients other clinical benefits, such as lower morbidity and shorter hospitalization time (11). Hence, RDP has broadened the indications.

In addition, previous studies have focused on all diseases including PDAC and other malignant lesions which is not recommended in our department. Therefore, the primary objective of our study was to share the experience in our institution that RDP could improve the SP rate compared to LDP among patients with benign and low-grade malignant distal lesions of the pancreas. We present the following article in accordance with the STROBE reporting checklist (available at http://dx. doi. org/10. 21037/tcr-192121).

\section{Methods}

\section{Patients and study design}

A total of 176 patients who underwent RDP or LDP at Changhai Hospital from January 2011 to October 2018 were included in this study. The patient treatment flow diagram is shown in Figure 1. The indications of RDP/ LDP for benign and low-grade malignant pathology are as follows: intraductal papillary mucinous neoplasm (IPMN); mucinous cystic neoplasm; symptomatic serous neoplasm; solid-pseudopapillary neoplasm; neuroendocrine neoplasm and pancreatitis. According to medical records (laboratory data and preoperative imaging findings), 67 patients received RDP while 109 patients were considered eligible for LDP. Both RDP and LDP were performed by experienced surgeons from the Department of Pancreatic Surgery, Changhai Hospital. Ten excluded cases were pancreatic ductal adenocarcinoma (PDAC). As a result, there were 63 cases of RDP and 103 cases of LDP. The clinicopathological and surgical outcomes were retrospectively compared between the two groups in an intention-to-treat analysis. The patients provided their informed consent before the surgery according to the rules and regulations of our institution. The study was conducted in accordance with the Declaration of Helsinki (as revised in 2013). Shanghai Changhai Hospital Ethics Committee approved the study and provided a certificate of exemption.

\section{Statistical analysis}

All variables are presented as the mean and standard deviation. Student's $t$-test was used when the data distribution was normal while the Mann-Whitney rank sum test was used for skewed variables. Proportions were compared using the Chi square test or Fisher's exact test. Logistic regression was used for correlation analysis. All analyses were performed using the SPSS 21.0 software program. A P value of less than 0.05 was considered the threshold for statistical significance.

\section{Results}

\section{Preoperative demographics and laboratory data}

Most of the data were comparable between the two groups. As shown in Table 1, there were no significant differences in age, sex, BMI, white blood cell count, serum hemoglobin level, platelet, serum albumin level, total bilirubin, alanine aminotransferase or CA-199 between the two groups. Taken together, the baseline preoperative characteristics between the RDP and LDP groups were well matched (all $\mathrm{P}$ value $>0.05$ ).

\section{Patient and clinicopathologic characteristics}

Consistent with other preoperative parameters, tumor size 
was comparable in the two groups $(3.93 \pm 2.66$ vs. $3.36 \pm 1.90$, $\mathrm{P}=0.150$, Table 2). In addition, the most common indications included in our study were symptomatic serous neoplasm (46\% vs. 40\%); solid-pseudopapillary neoplasm (22\% vs. $19 \%)$; intraductal papillary mucinous neoplasm (IPMN) (10\% vs. $13 \%)$; mucinous cystic neoplasm (11\% vs. $12 \%)$; neuroendocrine neoplasm ( $8 \%$ vs. $13 \%)$ and pancreatitis (3\% vs. $3 \%)$.

\section{Intraoperative variables and postoperative outcomes}

The key operative approach and intraoperative outcomes

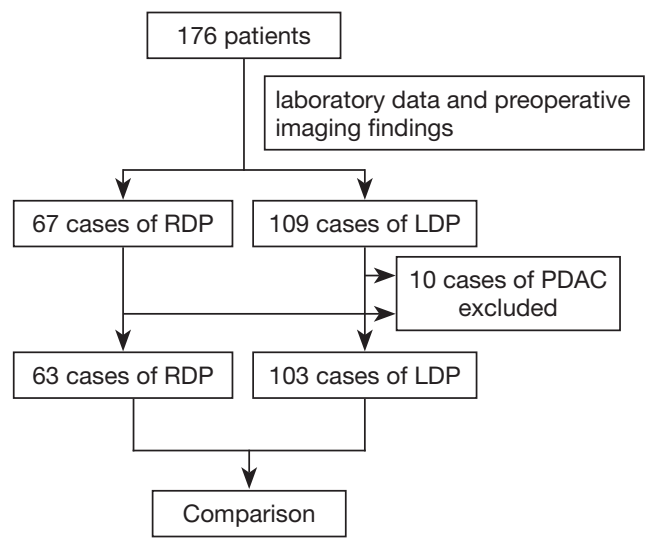

Figure 1 Flow chart of the patients. were not significantly different between the RDP and LDP groups with the exception of spleen preservation. The rate of spleen preservation in the RDP group (30.2\% vs. $6.8 \%, \mathrm{P}<0.001)$ was much higher. Additional intraoperative variables including operative time $(149.2 \pm 49.8$ vs. $150.0 \pm 50.2, \mathrm{P}=0.922)$ and blood loss $(167.3 \pm 166.7$ vs. $188.4 \pm 195.7, \mathrm{P}=0.481)$ were similar between both groups. On the other hand, 4 patients $(6.3 \%)$ in the RDP group needed a blood transfusion, while the rate in the LDP group was $12.6 \%$. Postoperative outcomes and complications are also listed in Table 3. There were also no deaths documented in the study group within 30 days after the operative procedure in both groups. According to the International Study Group (ISGPS) definition and grading of postoperative pancreatic fistula (POPF), the patients received POPF after surgery. Among these patients, grade A-type POPF occurred in 11 patients in the RDP group (27 patients in LDP group, $\mathrm{P}=0.193$ ), while grade $\mathrm{B}$-type POPF occurred in 3 patients (7 patients in the LDP group, $\mathrm{P}=0.843$ ). None of the patients developed a grade C-type POPF. These results were not significantly different. Moreover, these patients experienced full recovery without reoperation. As a result, there were also no significant differences in hospital stay $(6.0 \pm 3.0$ vs. $6.4 \pm 2.5, \mathrm{P}=0.404)$ between the RDP and LDP approaches while patients in RDP group needed to pay 30,000 RMB additional cost for the use of robot.

Table 1 Demographics and perioperative outcomes of patients

\begin{tabular}{|c|c|c|c|}
\hline Characteristics & RDP & LDP & $P$ value \\
\hline Sex (male/female) & $13 / 50$ & $25 / 78$ & 0.588 \\
\hline Age (years) & $44.5 \pm 14.9$ & $48.8 \pm 14.8$ & 0.086 \\
\hline BMI $\left(\mathrm{kg} / \mathrm{m}^{2}\right)$ & $22.8 \pm 3.47$ & $22.6 \pm 2.5$ & 0.256 \\
\hline $\mathrm{RBC}\left(\times 10^{12} / \mathrm{L}\right)$ & $4.6 \pm 0.6$ & $4.5 \pm 0.8$ & 0.298 \\
\hline HGB (g/L) & $142.4 \pm 14.4$ & $139.4 \pm 19.2$ & 0.278 \\
\hline PLT (×10%/L) & $150.2 \pm 68.7$ & $156.8 \pm 80.3$ & 0.590 \\
\hline TB $(\mu \mathrm{mol} / \mathrm{L})$ & $16.6 \pm 6.8$ & $16.0 \pm 7.6$ & 0.642 \\
\hline CA-199 (U/mL) & $21.8 \pm 19.6$ & $24.1 \pm 22.9$ & 0.497 \\
\hline
\end{tabular}

RDP, robot-assisted distal pancreatectomy; LDP, laparoscopic distal pancreatectomy. 


\section{Results}

We then separated patients into the SP group and splenectomy group to evaluate whether patients could benefit from SP and determine the reason for spleen

Table 2 Pathological outcomes

\begin{tabular}{lcc}
\hline Characteristics & RDP & LDP \\
\hline Total cases & 63 & 103 \\
Tumor size (cm) & $3.9 \pm 2.7$ & $3.4 \pm 1.9$ \\
Pathology (n) & & \\
IPMN & $6(10 \%)$ & $13(13 \%)$ \\
Mucinous cystic neoplasm & $7(11 \%)$ & $12(12 \%)$ \\
Symptomatic serous neoplasm & $29(46 \%)$ & $41(40 \%)$ \\
Solid-pseudopapillary neoplasm & $14(22 \%)$ & $20(19 \%)$ \\
Neuroendocrine neoplasm & $5(8 \%)$ & $14(13 \%)$ \\
$\begin{array}{l}\text { Pancreatitis } \\
\text { RDP, robot-assisted distal pancreatectomy; LDP, laparoscopic } \\
\text { distal pancreatectomy; IPMN, intraductal papillary mucinous } \\
\text { neoplasm. }\end{array}$ & $2(3 \%)$ & $3(3 \%)$ \\
\end{tabular}

preservation. As shown in Table 4, the SP and splenectomy groups were similar in patient characteristics and laboratory data, while patients in the SP group showed a smaller tumor size $(2.6 \pm 1.4$ vs. $3.9 \pm 2.4 \mathrm{~cm}, \mathrm{P}=0.005)$. However, there were no significant differences in operation time, blood loss, frequencies of pancreatic fistula or other postoperative variables, such as length of hospital stay. Furthermore, we analyzed the associations between clinical characteristics and SP using univariate logistic regression model. As demonstrated in Table 5, the univariate analysis confirmed that tumor size $(<2 \mathrm{~cm} / \geq 2 \mathrm{~cm})$ (hazard ratio HR, 7.255; 95\% CI, 2.269-23.198, $\mathrm{P}=0.001$ ) and surgery group (RDP/ LDP) (hazard ratio HR, 0.125; 95\% CI, 0.034-0.456, $\mathrm{P}=0.002$ ) were associated with SP rate. Besides, multivariate analysis showed that small tumor size and RDP were significantly associated with higher SP.

\section{Discussion}

In our study, the RDP group showed a significantly higher SP rate $(30.2 \%)$ than that of the LDP $(6.8 \%)$ group in patients with benign and low-grade malignant distal

Table 3 Postoperative outcomes and complications

\begin{tabular}{lccc}
\hline Characteristics & RDP & LDP & P value \\
\hline Total cases & 63 & 103 & 0.922 \\
Operative time (min) & $149.2 \pm 49.8$ & $150.0 \pm 50.2$ & 0.481 \\
Blood loss (mL) & $167.3 \pm 166.7$ & $188.4 \pm 195.7$ & 0.303 \\
Blood transfusion & $4(6.3 \%)$ & $13(12.6 \%)$ & $<0.001$ \\
Spleen-preserving & $19(30.2 \%)$ & $7(6.8 \%)$ & 1 \\
Converted to open (\%) & 0 & 1 & 1 \\
Mortality & 0 & 0 & 0.193 \\
POPF (n) & 11 & 27 & 0.843 \\
None or BL & 3 & 7 & 1 \\
B & 0 & 0 & 1 \\
C & 0 & 0 & 0.404 \\
Reoperation & $6.0 \pm 3.0$ & $6.4 \pm 2.5$ & 0.348 \\
Length of hospital stay (days) & $45,790.3$ & $47,456.5$ & 1 \\
Cost (RMB) & 30,000 & 0 & 0 \\
Additional cost (RMB) & 0 & 0 & 1 \\
Readmission & & 0 & 1 \\
\hline
\end{tabular}

RDP, robot-assisted distal pancreatectomy; LDP, laparoscopic distal pancreatectomy; POPF, postoperative pancreatic fistula. 
lesions of the pancreas, while there were no differences in other clinical variables. Besides, tumor size and RDP were dependent factors for SP.

With continuous developments in techniques, it is possible and safe to apply LDP for patients, which is reported to obviously improve patient recovery compared to traditional open surgery (12). However, LDP still has several technical limitations, such as reduced freedom of motion and narrowed two-dimensional visualization which may impact the surgery results $(6,13,14)$. Hence,

Table 4 Demographics and perioperative outcomes in SP group and non-SP group

\begin{tabular}{lccc}
\hline Characteristics & $\mathrm{SP}$ & $\mathrm{n}-\mathrm{SP}$ & $\mathrm{P}$ value \\
\hline Total cases & 26 & 140 & \\
Sex (male/female) & $4 / 22$ & $34 / 106$ & 0.461 \\
Age (years) & $44.1 \pm 14.4$ & $47.0 \pm 15.1$ & 0.260 \\
BMI $\left(\mathrm{kg} / \mathrm{m}^{2}\right)$ & $22.5 \pm 2.1$ & $22.8 \pm 3.2$ & 0.585 \\
WBC $\left(\times 10^{9} / \mathrm{L}\right)$ & $5.6 \pm 2.4$ & $5.2 \pm 2.0$ & 0.271 \\
RBC $\left(\times 10^{12} / \mathrm{L}\right)$ & $4.6 \pm 0.6$ & $4.6 \pm 0.7$ & 0.820 \\
HGB $(\mathrm{g} / \mathrm{L})$ & $137.1 \pm 14.8$ & $142.0 \pm 17.1$ & 0.082 \\
PLT $\left(\times 10^{9} / \mathrm{L}\right)$ & $144.1 \pm 59.8$ & $142.6 \pm 62.2$ & 0.888 \\
TB $(\mu \mathrm{mol} / \mathrm{L})$ & $16.6 \pm 6.6$ & $16.4 \pm 7.3$ & 0.900 \\
ALB $(\mathrm{g} / \mathrm{L})$ & $41.8 \pm 4.3$ & $42.5 \pm 4.5$ & 0.362 \\
ALT $(\mathrm{U} / \mathrm{L})$ & $38.0 \pm 33.3$ & $34.6 \pm 33.1$ & 0.531 \\
$\mathrm{CA}-199(\mathrm{U} / \mathrm{mL})$ & $18.5 \pm 12.0$ & $23.9 \pm 22.7$ & 0.129 \\
Tumor size $(\mathrm{cm})$ & $2.6 \pm 1.4$ & $3.9 \pm 2.4$ & 0.005 \\
\hline
\end{tabular}

SP, spleen preservation. robot-assisted surgery has developed to overcome these limitations with increased freedom and improved threedimensional visualization. Because a small rupture of the splenic vessels can necessitate splenectomy (15-18), robotassisted surgery is reported to greatly benefit patients, especially for improving SP rates $(11,19,20)$. Furthermore, spleen preservation is highly recommended due to its advantages for immunity and hematopoiesis for patients with nonmalignant tumors (21).

Here, we retrospectively analyzed 166 patients in our institution, including 63 cases of RDP and 103 cases of LDP. Our results demonstrated that RDP improved SP rates in patients with benign and low-grade malignant distal lesions of the pancreas. Several key points in our surgical improvements were introduced as follows. First, as the PV is sometimes close to the pancreas or even growing in the distal of the pancreas, the spleen vein should be exposed at the pancreatic neck. We can dissect SMV and then find the PV along the SMV. This point can reduce the damage to IMV. Second, we can ensure that the PA is exposed on the upper edge of the pancreas before the pancreas is cut off. This procedure can reduce the damage to the PA. Third, we can fully dissect the lower margin of the pancreas to the spleen, which is beneficial for surgeons to handle accidental bleeding. Finally, the ligaments between the spleen and stomach can be reserved as much as possible to preserve the feasibility of implementing the Warshaw procedure. In conclusion, due to improved three-dimensional visualization and the advantages of suturing and knotting, surgeons can dissect vessels precisely and control excessive bleeding in a timely manner, which might preserve spleen in patients with appropriate tumor sizes.

Table 5 Univariate and multivariate analyses of factors associated with spleen preservation

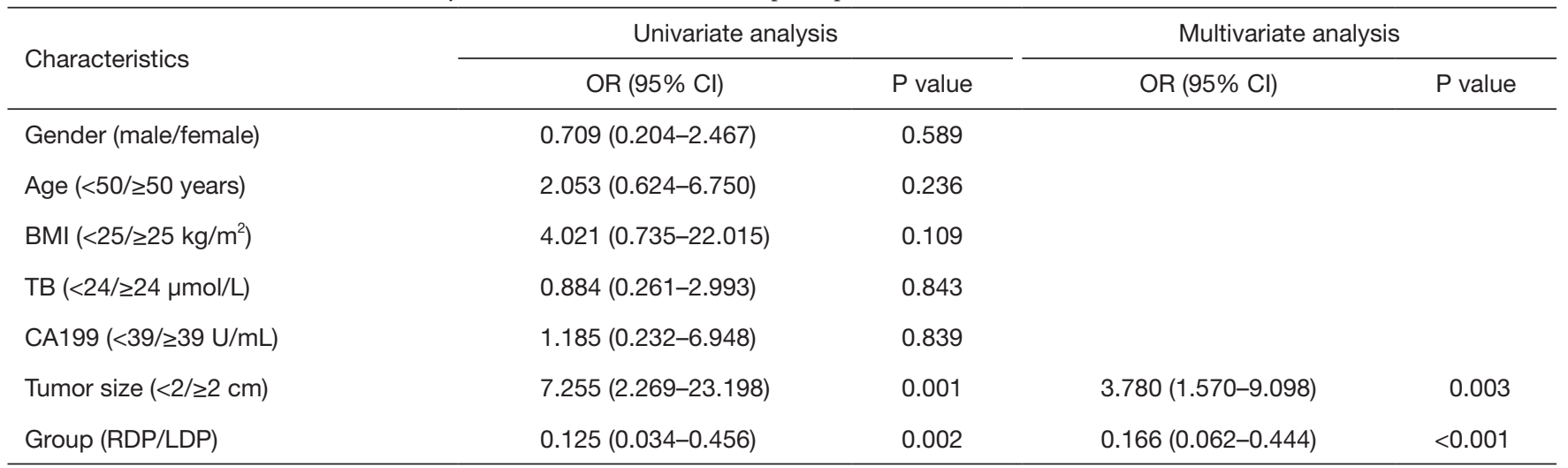

RDP, robot-assisted distal pancreatectomy; LDP, laparoscopic distal pancreatectomy. 
It is obvious that the RDP approach has great advantages over the LDP approach in distal pancreatectomy. However, the difficulty of the RDP approach includes a learning curve and rich experience in open surgery. In addition, recent meta-analyses have demonstrated that the mean operation time in RDP and LDP was 247.8 and 229.9 min, respectively (22). Our results showed that the RDP group was not associated with longer operative times (149.2 vs. $150.0 \mathrm{~min}$ ), which were much shorter than reported, because of accumulated surgical experience in minimally invasive techniques. Furthermore, RDP has obvious advantages in the invasion of large benign tumors into blood vessels, especially when repairing ruptured $\mathrm{PV}$ and $\mathrm{PA}$. Bleeding control is one of the primary advantages of the robotic technique and previous studies have indicated that RDP resulted in less blood loss $(9,10)$. In our study, there was no difference in blood loss (167.3 vs. $188.4 \mathrm{~mL}$ ) between the two groups. Consistent with a recent study, RDP did not decrease the occurrence or severity of pancreatic fistula (23), or the severity of the pancreatic fistula rate. Notably, the POPF rate in our patients $(22 \%$ in RDP group and $33 \%$ in LDP group) was also increased higher than reported (3\% in RDP group and $12 \%$ in LDP group). The reason for the higher POPF rate is that the samples for the study are mostly benign or low-grade malignant lesions of pancreas. Their pancreatic secretion function is normal, and their structures are soft. In consideration of the "learning curve", we anticipate that the RDP technique would improve bleeding control and reduce the rate of pancreatic fistula compared in a further study.

As a retrospectively study, it certainly has its limitations. As all patient demographics as well as intra- and postoperative outcomes were retrospectively collected, inherent selection bias is inevitable. Additionally, patients in RDP group are included since the robotic surgery is available in our center, so the learning curve with robot technology may influence the comparison results. In addition, as the financial burden of robotic procedures is reduced, we may conduct a randomized controlled trial to investigate the benefits of RDP over LDP.

In conclusion, our study demonstrates that RDP has comparable safety and efficacy to LDP for patients with benign and low-grade malignant lesions of the pancreas. With the advantages of the robot technique, surgeons can benefit patients greatly, especially for high SP rates, while high-grade malignant lesions of the pancreas are not recommended in our institutions.

\section{Acknowledgments}

Funding: The National Natural Science Foundation of China (No: 81572862) and Shanghai "Rising Stars of Medical Talent" Youth Development financially supported the design, collection, analysis and writing of the manuscript.

\section{Footnote}

Reporting Checklist: The authors have completed the STROBE reporting checklist. Available at http://dx.doi. org/10.21037/tcr-19-2121

Data Sharing Statement: available at http://dx. doi. org/10. 21037/tcr-19-2121

Conflicts of Interest: All authors have completed the ICMJE uniform disclosure form (available at http://dx. doi. org/10.21037/tcr-19-2121). The authors have no conflicts of interest to declare.

Ethical Statement: The authors are accountable for all aspects of the work in ensuring that questions related to the accuracy or integrity of any part of the work are appropriately investigated and resolved. All patients provided a written informed consent. The study was conducted in accordance with the Declaration of Helsinki (as revised in 2013). Shanghai Changhai Hospital Ethics Committee approved the study and provided a certificate of exemption.

Open Access Statement: This is an Open Access article distributed in accordance with the Creative Commons Attribution-NonCommercial-NoDerivs 4.0 International License (CC BY-NC-ND 4.0), which permits the noncommercial replication and distribution of the article with the strict proviso that no changes or edits are made and the original work is properly cited (including links to both the formal publication through the relevant DOI and the license). See: https://creativecommons.org/licenses/by-nc-nd/4.0/.

\section{References}

1. Klompmaker S, van der Vliet WJ, Thoolen SJ, et al. Procedure-specific Training for Robot-assisted Distal Pancreatectomy. Ann Surg 2019. [Epub ahead of print].

2. Pais-Costa SR, Sousa GCC, Araujo SLM, et al. 
Laparoscopic distal pancreatectomy with spleen preservation. Arq Bras Cir Dig 2018;31:e1395.

3. Pais-Costa SR, Sousa GCC. Laparoscopic distal pancreatectomy with or without spleen preservation: comparative analysis of short and long-term outcomes. Arq Bras Cir Dig 2019;32:e1461.

4. Zhao W, Liu C, Li S, et al. Safety and efficacy for robotassisted versus open pancreaticoduodenectomy and distal pancreatectomy: A systematic review and meta-analysis. Surg Oncol 2018;27:468-78.

5. Alfieri S, Boggi U, Butturini G, et al. Full Robotic Distal Pancreatectomy: Safety and Feasibility Analysis of a Multicenter Cohort of 236 Patients. Surg Innov 2020;27:11-8.

6. Najafi N, Mintziras I, Wiese D, et al. A retrospective comparison of robotic versus laparoscopic distal resection and enucleation for potentially benign pancreatic neoplasms. Surg Today 2020;50:872-80.

7. Quero G, Fiorillo C, Alfieri S. Distal pancreatectomy in the new era of minimally invasive surgery: the on-going debate on the cost-effectiveness. Hepatobiliary Surg Nutr 2019;8:659-61.

8. Sahara K, Tsilimigras DI, Moro A, et al. Long-Term Outcomes after Spleen-Preserving Distal Pancreatectomy for Pancreatic Neuroendocrine Tumors: Results from the US Neuroendocrine Study Group. Neuroendocrinology 2020. [Epub ahead of print].

9. Hu YH, Qin YF, Yu DD, et al. Meta-analysis of short-term outcomes comparing robot-assisted and laparoscopic distal pancreatectomy. J Comp Eff Res 2020;9:201-18.

10. Liu R, Liu Q, Zhao ZM, et al. Robotic versus laparoscopic distal pancreatectomy: A propensity score-matched study. J Surg Oncol 2017;116:461-9.

11. Alfieri S, Butturini G, Boggi U, et al. Short-term and longterm outcomes after robot-assisted versus laparoscopic distal pancreatectomy for pancreatic neuroendocrine tumors (pNETs): a multicenter comparative study. 2019;404:459-68.

12. Ohtsuka T, Nagakawa $\mathrm{Y}$, Toyama H, et al. A multicenter prospective registration study on laparoscopic pancreatectomy in Japan: report on the assessment of 1,429 patients. J Hepatobiliary Pancreat Sci 2020;27:47-55.

13. Sugimachi K, Iguchi T, Ohta M, et al. Laparoscopic spleen-preserving distal pancreatectomy for a solid-cystic intraabdominal desmoid tumor at a gastro-pancreatic lesion: a case report. BMC Surg 2020;20:24.

14. Vicente E, Nunez-Alfonsel J. A cost-effectiveness analysis of robotic versus laparoscopic distal pancreatectomy. Int J Med Robot 2020;16:e2080.

15. Hernandez MC, Khasawneh M, Contreras-Peraza N, et al. Vaccination and splenectomy in Olmsted County. Surgery 2019;166:556-63.

16. Kurata N, Ogura Y, Ogiso S, et al. Splenectomy in living donor liver transplantation and risk factors of portal vein thrombosis. Hepatobiliary Pancreat Dis Int 2019;18:337-42.

17. Phipps WE, de Laforcade AM, Barton BA, et al. Postoperative thrombocytosis and thromboelastographic evidence of hypercoagulability in dogs undergoing splenectomy for splenic masses. J Am Vet Med Assoc 2020;256:85-92.

18. Yohanathan L, Loveday BPT, Brar N, et al. Effect of vessel preservation on splenic volume and function in patients with spleen preserving distal pancreatectomies. HPB (Oxford) 2020;S1365-182X(20)30031-9.

19. Souche R, Herrero A, Bourel G, et al. Robotic versus laparoscopic distal pancreatectomy: a French prospective single-center experience and cost-effectiveness analysis. Surg Endosc 2018;32:3562-9.

20. Xu SB, Jia CK, Wang JR, et al. Do patients benefit more from robot assisted approach than conventional laparoscopic distal pancreatectomy? A meta-analysis of perioperative and economic outcomes. J Formos Med Assoc 2019;118:268-78.

21. Pendola F, Gadde R, Ripat C, et al. Distal pancreatectomy for benign and low grade malignant tumors: Shortterm postoperative outcomes of spleen preservation-A systematic review and update meta-analysis. J Surg Oncol 2017;115:137-43.

22. Zhou JY, Xin C, Mou YP, et al. Robotic versus Laparoscopic Distal Pancreatectomy: A Meta-Analysis of Short-Term Outcomes. PLoS One 2016;11:e0151189.

23. Gavriilidis P, Lim C, Menahem B, et al. Robotic versus laparoscopic distal pancreatectomy - The first metaanalysis. HPB (Oxford) 2016;18:567-74.

Cite this article as: Jiang $\mathrm{Y}$, Zheng $\mathrm{K}$, Zhang $\mathrm{S}$, Shao $\mathrm{Z}$, Cheng P, Zhang Y, Jin G, He T. Robot-assisted distal pancreatectomy improves spleen preservation rate versus laparoscopic distal pancreatectomy for benign and lowgrade malignant lesions of the pancreas. Transl Cancer Res 2020;9(9):5166-5172. doi: 10.21037/tcr-19-2121 